\section{PWE-172 A NATIONAL BIOFEEDBACK PRACTITIONERS SERVICE EVALUATION}

${ }^{1} \mathrm{KJ}$ Etherson*, ${ }^{2} \mathrm{E}$ Horrocks, ${ }^{2} \mathrm{M}$ Scott, ${ }^{2} \mathrm{CH}$ Knowles, ${ }^{1} \mathrm{Y}$ Yiannakou. ${ }^{1}$ Colorectal Surgery, County Durham and Darlington NHS Foundation Trust, Durham, UK; ${ }^{2}$ National Centre for Bowel Research and Surgical Innovation, Queen Mary University London, London, UK

\subsection{6/gutjnl-2014-307263.432}

Introduction Within the UK, there is anecdotal evidence of disparity in the training, practice, supervision, and perception of efficacy amongst practitioners of biofeedback therapy for chronic constipation.

Methods Between October 2012 and October 2013, a prospective service evaluation was distributed to biofeedback practitioners in the UK through academic conferences or by invitation to complete an online assessment form.

Results 76 practitioners responded, consisting of nurses (47\%), physiotherapists (35\%), physiologists (12\%) and others (7\%). Only 45\% described 'biofeedback' consistent with an accepted definition. $86 \%$ use equipment to provide sensory feedback. Methods of biofeedback varied: balloon catheter (54\%), brace pump technique $(78 \%)$, urge resistance $(83 \%)$, irrigation (16\%), and relaxation (12\%). Only $65 \%$ of practitioners had attended formal training courses, and 52\% considered themselves to be self-tau, UKght. $36 \%$ receive formal supervision and only $38 \%$ of those by a senior. Regular audit of outcomes is undertaken by $67 \%$. UK-wide perception of treatment response for chronic constipation is markedly variable (mean response $=57 \%[\mathrm{IQR}$ : $50-75 \%$, SD 23\%]); there were no differences in perception of treatment response between nurses or physiotherapists. Practitioners' free responses demonstrated strong positive themes of a holistic approach and an overall perception of effectiveness. Negative themes included service restrictions.

Conclusion There is marked variation in practice, training, and supervision of biofeedback therapists throughout the UK. Perceptions of efficacy vary greatly. Development of training and supervision standards is a priority as well as consensus to standardise therapy.

Disclosure of Interest None Declared.

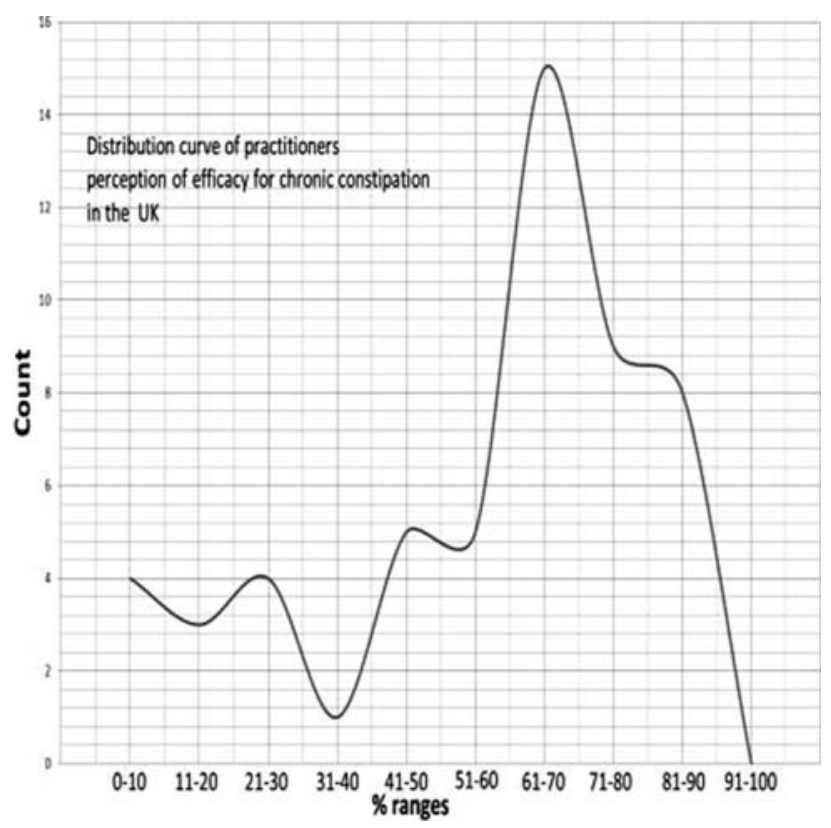

Abstract PWE-172 Figure 1
PWE-173 PERIPHERAL AND CENTRAL EFFECTS OF A SINGLE DOSE OF THE GLUCAGON-LIKE PEPTIDE-1 RECEPTOR AGONIST EXENATIDE

${ }^{1} \mathrm{~L}$ Wasse ${ }^{*},{ }^{2} \mathrm{~T}$ Little, ${ }^{3} \mathrm{~T}$ Coulson, ${ }^{1} \mathrm{C}$ Bryant, ${ }^{4} \mathrm{~S}$ McKie, ${ }^{1} \mathrm{~J}$ McLaughlin. ${ }^{1}$ Institute of Inflammation and Repair, University of Manchester, Manchester, UK; ${ }^{2}$ Boden Institute of Obesity, Nutrition, Exercise and Eating Disorders, University of Sydney, Sydney, Australia; ${ }^{3}$ Salford Royal NHS Foundation Trust, Salford, UK; ${ }^{4}$ Institute of Brain, Behaviour and Mental Health, University of Manchester, Manchester, UK

\subsection{6/gutjnl-2014-307263.433}

Introduction Administration of the glucagon-like peptide-1 receptor agonist (GLP-1RA) exenatide reduces food intake and induces weight loss. GLP-1 receptors are located throughout the body including the brain and vagal afferent neurones. Although exenatide slows gastric emptying (GE), it is believed the main mechanism behind exenatide-induced weight loss may be related to activation of central pathways that mediate satiety. However, research in humans is lacking. Brain activation can be indirectly measured by quantifying changes in the blood oxygen level dependent (BOLD) signal measured during functional magnetic resonance imaging (fMRI).

Methods To confirm a gastric inhibitory effect of exenatide, 12 healthy volunteers received a subcutaneous injection of $5 \mu \mathrm{g}$ exenatide or placebo on separate occasions $60 \mathrm{~min}$ prior to consumption of a $0.5 \mathrm{M}$ carbohydrate test drink $(500 \mathrm{~mL})$. Over 45 min GE was non-invasively measured using breath tests. Then, to examine brain activation using fMRI, 15 healthy volunteers received $5 \mu \mathrm{g}$ exenatide or placebo after $\sim 10 \mathrm{~h}$ fast and underwent 70 min scanning (3T Philips scanner). Wholebrain analysis was conducted (data for the hypothalamus only are reported). For each volunteer the difference in mean\%BOLD signal change from baseline between exenatide and placebo was extracted and analysed in $5 \mathrm{~min}$ blocks using T-tests. Two volunteers were excluded due to excessive movement during scans.

Results Repeated measures ANOVA revealed that exenatide significantly slowed GE of the test drink with the area under the curve for exenatide being 31\% lower than placebo ( $\mathrm{p}<0.005$ ). fMRI analysis revealed bilateral increases in BOLD signal in the lower hypothalamus $(\mathrm{p}<0.05)$, and a trend for increased BOLD $(\mathrm{p}<0.075)$ in the right upper hypothalamus $45 \mathrm{~min}$ after exenatide. Conversely, BOLD signal was decreased in the left upper hypothalamus 40min after exenatide ( $\mathrm{p}<0.05)$.

Conclusion Our data confirms that a single dose of exenatide potently delays GE. Furthermore, most regions of the hypothalamus, a key appetite regulatory brain area, were progressively activated $45 \mathrm{~min}$ after exenatide administration. Those volunteers were fasted which suggests that the anorectic effects of exenatide in humans could be mediated via activation of central GLP-1 receptors, either directly or via vagal inputs. Further analysis will now be conducted to examine the effect of exenatide on areas in the brain associated with food reward such as the insula and orbitofrontal cortex.

Disclosure of Interest None Declared.

\section{PWE-174 A PROOF OF CONCEPT ASSESSMENT OF NON-INVASIVE VAGUS NERVE STIMULATION (NVNS) WITH GAMMACORE $®$ IN PATIENTS WITH GASTROPARESIS AWAITING ENTERRA® IMPLANTATION}

MF Jaboli*, J Bennell, O Epstein. Gastroenterology, Royal Free Hospital, London, UK 
BSG 2014 abstracts

\begin{tabular}{|c|c|c|c|}
\hline \multicolumn{4}{|l|}{ Symptoms } \\
\hline (7 patients) & Mean pre-treatment score (range) & Mean score in final two weeks (range) & Percent change \\
\hline Composite & $34.7(18.3-62.4)$ & $27.49(14.7-59.3)$ & $-21 \%$ \\
\hline Nausea & $2.8(0.6-4.7)$ & $2.2(0.5-4.3)$ & $-21 \%$ \\
\hline Vomiting & $1.2(0-5)$ & $1.2(0.1-5)$ & $0 \%$ \\
\hline Early satiety & $3.3(2.1-5)$ & $2.6(1.7-5)$ & $-21 \%$ \\
\hline Bloating & $2.4(1.1-3.5)$ & $1.7(0.4-3.2)$ & $-29 \%$ \\
\hline
\end{tabular}

Introduction Vagal dysfunction has been implicated in gastroparesis. Gut vagal afferents convey symptoms of nausea, bloating and early satiety but the nerve also has an antinociceptive function. GammaCore (electroCore, LLC: New Jersey) is a CE marked hand-held vagus nerve stimulator designed to selectively stimulate afferent vagal A-fibres. It is possible that in gastroparesis, stimulation of the vagus nerve as it traverses the neck might influence symptoms.

Methods Fifteen patients with severe gastroparesis awaiting Enterra (Medtronic, Minnesota) implantation agreed to a short proof of concept assessment of nVNS. Each patient was supplied with a GammaCore device programmed to deliver 150 doses, each dose lasting 90 seconds. The electrodes on the GammaCore device were positioned in line with the right cervical vagus nerve and stimulation applied three times daily. The gastroparesis multi-symptom questionnaire, that includes the symptoms of nausea, vomiting, early satiety and bloating, was completed daily for the week prior to starting treatment and daily throughout the treatment period. Symptoms were scored on a Likert scale $(1=$ none and $5=$ severe $)$. Composite and individual symptom scores were summated for the week preceding treatment and the final two weeks of the treatment period.

Results Only seven of the 15 patients complied with the treatment regimen. In six patients, the diary score cards were incomplete and two patients did not use the GammaCore.

Conclusion In this group of severely ill patients awaiting Enterra implantation, half complied with the treatment regimen. Compliant patients scored improvement in nausea, early satiety and bloating, as well as the composite gastroparesis score. This short term proof of concept assessment suggests that nVNS influences symptoms conveyed by vagal afferents. It is possible that stimulation of both left and right vagus nerves, increased stimulation amplitude, and a longer period of treatment might improve responsiveness. Future clinical trials are warranted to elucidate safety, efficacy, dose response and compliance.

Disclosure of Interest None Declared.

\section{PWE-175 COST-EFFECTIVENESS OF LINACLOTIDE (LIN) COMPARED TO ANTIDEPRESSANTS (ATDS) IN THE TREATMENT OF IRRITABLE BOWEL SYNDROME WITH CONSTIPATION (IBS-C) IN SCOTLAND}

${ }^{1} \mathrm{M}$ Fisher, ${ }^{2} \mathrm{~A}$ Walker, ${ }^{3} \mathrm{M}$ Falqués, ${ }^{4} \mathrm{M}$ Rance*, ${ }^{5} \mathrm{D}$ Taylor, ${ }^{3} \mathrm{~L}$ Lindner. ${ }^{1} W G$ Consulting, High Wycombe, UK; ${ }^{2}$ Glasgow University, Glasgow, UK; ${ }^{3}$ Almirall S. A., Barcelona, Spain; ${ }^{4}$ Almirall UK, Uxbridge, UK; ${ }^{5}$ Ironwood Pharmaceuticals, Cambridge, Massachusetts, USA

\subsection{6/gutjnl-2014-307263.435}

Introduction IBS-C is a chronic functional gastrointestinal disorder that has been shown to decrease quality of life (QoL) and work productivity, and increase activity impairment. LIN is indicated by the European Medicines Agency as the only treatment for IBS-C (covering all key symptoms) in adults and has received a positive recommendation from the Scottish Medicines
Consortium. This study sought to determine the cost-effectiveness of LIN compared with ATDs for the treatment of adults with moderate to severe IBS-C who have previously received antispasmodics and/or laxatives.

Methods A Markov model was created to estimate costs and quality-adjusted life years (QALYs) on 4-week cycles over a 5-year time horizon from the perspective of NHS Scotland. The comparator used was ATDs, reflecting Scottish current practice. Health states were based on treatment satisfaction (satisfied, moderately satisfied, not satisfied) and death. Transitions between states were based on satisfaction data from the LIN pivotal studies and the Scottish general all-cause mortality statistics. Treatment costs were calculated from the British National Formulary using the least-expensive ATD cost (amitriptyline hydrochloride $20 \mathrm{mg}$ ). NHS resource use for each state was collected from Scottish clinician interviews and combined with NHS Reference costs to obtain disease-related costs. QoL was based on the EQ-5D collected from the pivotal studies. Uncertainty was explored through extensive sensitivity analyses.

Results The base case comparing LIN with ATDs over 5 years provided an incremental cost-effectiveness ratio (ICER) value (cost per QALY) of $£ 7,370$. Scenario analyses conducted to address structural uncertainty resulted in ICERs ranging from $£ 1,204$ to $£ 10,899$. Probabilistic sensitivity analysis was also conducted using 10,000 iterations to examine parameter uncertainty over the entire model. This analysis showed that there was an $83 \%$ probability that LIN was cost-effective at a willingness to pay an ICER of $£ 20,000$ when compared with ATDs. The mean probabilistic ICER was $£ 4,606$ (mean incremental costs $£ 394$ and mean incremental QALYs 0.085).

Conclusion LIN is a cost-effective treatment for adults with moderate to severe IBS-C who have previously received antispasmodics and/or laxatives. The ICER in Scotland was calculated as $£ 7,370$ when compared with ATDs and was based on conservative assumptions. Results were robust to extensive sensitivity analyses.

Study funded by Almirall UK.

Disclosure of Interest M. Fisher Conflict with: former employee of WG Consulting, A. Walker Consultant for: WG Consulting, Conflict with: WG Consulting (Advisory Board meeting), M. Falqués Employee of: Almirall S. A., M. Rance Employee of: Almirall UK, D. Taylor Employee of: Ironwood Pharmaceuticals, L. Lindner Employee of: Almirall S. A.

\section{PWE-176 PREVALENCE, MANAGEMENT, AND HEALTHCARE BURDEN OF IRRITABLE BOWEL SYNDROME (IBS) IN SCOTLAND}

${ }^{1} \mathrm{~S}$ McTaggart, ${ }^{1} \mathrm{G}$ Wyper, ${ }^{1} \mathrm{~L}$ Harkins, ${ }^{1} \mathrm{I}$ Bishop, ${ }^{1} \mathrm{M}$ Bennie ${ }^{1},{ }^{2} \mathrm{M}$ Rance*. ${ }^{1} \mathrm{NHS}$ National Services UK, Edinburgh, UK; ${ }^{2}$ Almirall, Uxbridge, UK

\subsection{6/gutjnl-2014-307263.436}

Introduction Using real-world healthcare, routinely collected information we investigated the prevalence of irritable bowel 